\title{
Design and Implementation of Simulation Maintenance Training System for Aircraft Engine Electrical System
}

\author{
Zheng Wang ${ }^{a}$, Xingdong Zhu, Jiali Fan, Jianhua Song \\ Naval Aviation Engineering Institute-Qingdao Branch Qingdao China \\ aemail:w_zheng@public.qd.sd.cn
}

Keywords: Aircraft engine; Electrical system; Maintenance training; Fault simulation

\begin{abstract}
Since the theory of aircraft engine electrical system was more complicated and difficult to master for maintenance support personnel, a novel scheme of simulation system design for maintenance training was proposed. The mechanical frame of the system was based on the original aircraft engine and electrical parts of the engine were reconstructed by DSP digital technology. As the core of communication the integrated console based on embedded Linux was developed under Eclipse environment. The experimental equipment environment with virtue simulation and strong interaction was constituted by employing the electric network technology. The system realized the functions such as comprising theory teaching, state simulation, fault setting and training testing with electrical network technology.
\end{abstract}

\section{Introduction}

Some problems such as very difficult to trouble shooting, to mastering the principle were presented universally in maintenance support, because of the control process and control principle becoming more complex along with the increasing of functions and components for aircraft engine electrical system. In order to troubleshooting the fault rapidly and exactly, the maintenance supporter must be trained with real equipments to master the skills. There are very large limitation in operation of training with equipments, because of some training item with devastating to shorten the life of component. By using digital control technology, bus network technology, electric and electron technology, the aircraft engine electrical system maintenance training simulation system was designed according to requirement of equipment support training. The system can realize the functions such as assisting teaching, status simulation, troubleshooting training, test and evaluation. It can improve effectiveness of training and decrease the training funds.

\section{Overall Design of the Simulation Maintenance Training System}

\section{A. Overall Design Scheme}

Simulation maintenance training system for engine electrical system was constituted by two parts: the console and engine model. The entire system was connected via multiple -bus, integrated console as the core, the overall connection relationship shown in Figure 1.

As the core of system, the integrated control console was constituted by the display and control terminals, control panel and the frequency conversion controller. Display and control terminal as a interactive window for monitoring various engine electrical system status and setting fault, use the embedded Linux operating system to meet the demand of the system starting fast, convenient software development [1]. In order to simulate operation of the engine electrical system better, the frequency inverter was employed to simulate electrical characteristics of engine, so the speed switch for frequency inverter, speed knob and power button for main compnent of training system were set in the control panel. These switches, buttons and knobs realized the system control function. 


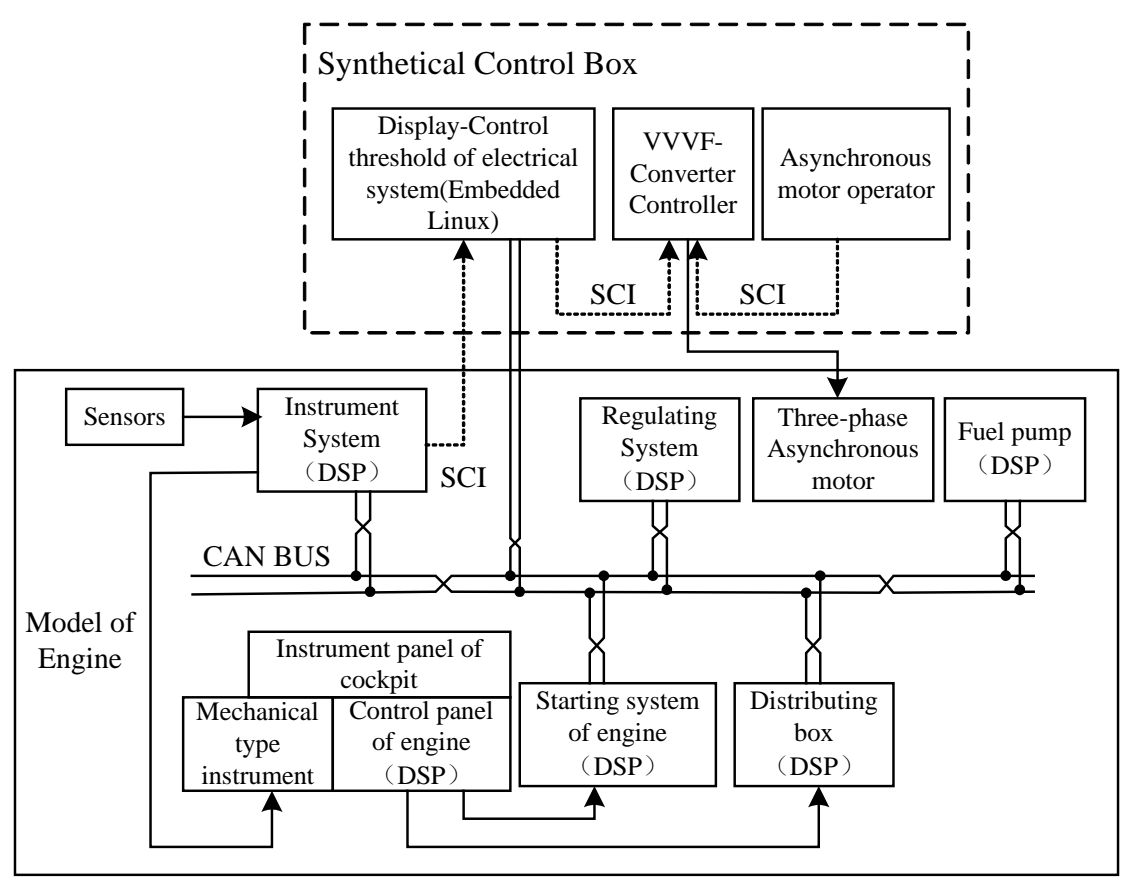

Figure 1. Structure Diagram of the System

The model engine simulated a certain type of aircraft engine. The electrical components and the necessary control equipment were installed in its internal. The complete aircraft engine electrical system was built. Model engine electrical components comprised: cockpit instrument panel, cockpit switchboards, motor control board cockpit, electronic engine controller, starter motor, fuel pump, etc. In order to enable electrical components to meet the control requirements, and the ability to share information using a communication network, the DSP were employed as control core for every component, and its internal digital transformation designed to maintain its dimensions, mounting positions and interface design relationship intact. In addition, the three-phase asynchronous motor was installed to simulate the real engine rotation.

After redesigned, each electrical subsystem with CAN bus and SCI communication line to communicate with integrated console, constituted an integrated aircraft engine maintenance training simulation system based on multi-bus.

\section{B. Function of the system}

The simulation maintenance training system for electrical system of aircraft engine covered two parts of aircraft engine electrical system maintenance skills training and theory teaching. The main function included theory teaching, fault set, state simulation and evaluation, and other examinations.

1) Teaching function

Within an integrated display and control console terminal storing electrical equipment and electronic tutorial lesson plans, the basic principle of each electrical system through text, graphics, video and other multimedia information presentation tools, training and demonstration structures and operational use of knowledge.

\section{2) state simulation}

The system can realize the inverter integrated console interoperation with the VVVF and all electrical components. The virtual instrument and switch status in display and control terminals can be reproduced in real time on the dynamic change in the engine control. It can also be targeted to the underlying state control of the subsystem.

3) failure settings

By integrated display and control console terminal, settings fault injection instructions was sent to the bottom of the respective electrical system components via CAN bus communication network to achieve fault setting. In this case, not only can actually see the outward manifestation of a fault occurred on the model of the engine, but also use instruments measuring circuit parameters, in-depth analysis of the causes of failure. 


\section{4) Examination Evaluation}

The system with a test mode can provide curriculum management, score management, paper management modules. Aspects of the question text, the topic rules, share scores and answer time can be updated. teachers can organize the papers according to a flexible teaching content, at any time the students take the exam papers and scores, checking teaching effect.

\section{Key Technology of Maintenance Training Simulation System}

\section{A. Simulation of aircraft engine based VVVF}

The aircraft engine model is simulated by a three-phase asynchronous motor of 22KW. The actual speed of the motor was the reaction speed of the engine. Three-phase induction motor speed control by the frequency controller, a control signal by the inverter controller integrated console through SCI communication line was given, the simulation process shown in Figure 2. Depending on the instruction, the simulation can be divided into the car and manual operation manual operation.

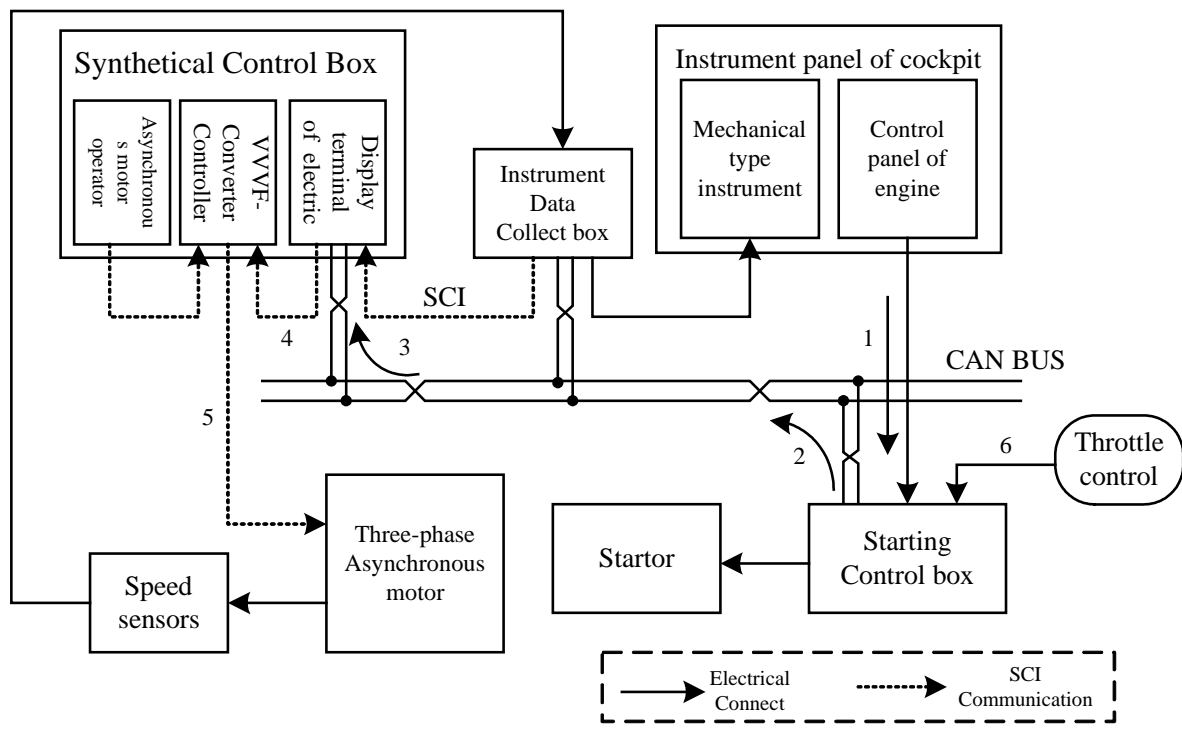

Figure 2. Work flow diagram of engine used VVVF simulation

In Figure 2, according to the order of $1 \rightarrow 6$, the start control box detects the pilot's instrument panel start button action, send a signal to the CAN bus, display and control terminal received this signal through the SCI communication line control three-phase inverter induction motor starting and maintaining idle state. In this state, the use of the throttle lever in the cockpit controlled three-phase induction motor. Speed control principle was when the throttle moves through the drive gear linkage rotary potentiometer, rotary potentiometer variable transmission signals generated by the control box to start, start the control box after $\mathrm{AD}$ conversion being transmitted via the CAN bus terminal display and control, display and control terminal of the drive through a three-phase induction motor control, ie $2 \rightarrow 6$ of repetitive movements.

\section{B. Systems management based on Eclipse platform}

With the development of equipment, training systems also need to be updated, which requires the system architecture and the content of the training requires a certain degree of scalability, and proposed for this article uses a simulation-based training system development program Eclipse platform.

Eclipse was an open source-based integrated development environment plug-ins, in addition to micro-kernel runtime platform outside. its all functions were implemented in the form of plug-in ${ }^{[2-4]}$.

Platform provided PDE (Plug-in development environment) for developers to create, test, debug, and deploy various types of development Eclipse plug-ins. When functional requirements can be developed in accordance with extension points Eclipse platform and the actual demand for new 
features widget, complete system upgrade update. Further Eclipse platform can request notifications for different types of events, as well as create a new view and so on.

According to general structure simulate maintenance training system, combined with Eclipse's extension mechanism is designed to simulate the Eclipse platform-based maintenance training system management side.

The objective was to design management for the development of end maintenance training simulation system to do an integrated development environment. By writing Eclipse plug-ins, integrated development management side maintenance training simulation system for the utility. Management side was designed based on the Eclipse development platform, through the development of Eclipse plug-ins to extend Eclipse target platform, simulation training systems necessary for the program development services. Through the preparation of specific training system virtual instruments, in response to the hot zone, data communications, and other functions Graphics Library interface, to achieve a custom application integration development environment. On this basis, and then expanded the instrumentation, electric start, voltage regulators, transmission and distribution, and other user interface, these user interface with menu options and navigation buttons and other interactive features.

\section{Fault settings Based on electronic switch circuit}

Aircraft engines rely mainly on power distribution control relays, contactors, switches and other machinery to achieve the mechanical switch does not have a distribution branch operating status information acquisition, processing and transmission capabilities, programmable control can not be loaded according to the requirements set by the fault. The system uses to replace the original relays, contactors and other electronic-based programmable intelligent switch, power distribution control, while achieving complete fault simulation capabilities. Electronic switch module uses intelligent power switch BTS555 supplemented by the corresponding drive control circuit, instead of the traditional relays, contactors and other power distribution control device to achieve real-time detection of programmable control and distribution branch status. DSP-based distribution control unit with real-time acquisition and processing of a plurality of branch distribution status information, the power distribution control unit via CAN bus interface to communicate with the network training system hook fault setting instruction in response to display and control terminals, control electronic switch output state to make it consistent with the time of the failure, failure to achieve the purpose set.

\section{Data exchange based on CAN network}

Training system included many electrical systems, and with different functions, various types, in order to realize system status monitoring, comprehensive control, particularly to achieve fault simulation capabilities, large data must be transferred. Because of large data traffic, and the requirement to reliability and reality, large information transformation was needed. Considering the size of the application, performance requirements and development costs and other factors, select the CAN bus simulation system maintenance to build a data communications network ${ }^{[5]}$.

Reliability of bus communication is directly related to system performance. In order to ensure the engine information transferred in real time, accreting and reliable transmission of all kinds of information to make normative design. Multi-bus communication scheme was employed. The need for timely, uninterrupted transmission of meter data to an integrated console for the virtual computer instruments, real-time demanding, so the meter data acquisition subsystem and the display and control communications between terminals using SCI serial bus. This also can reduce CAN bus traffic and avoid congestion CAN bus data, improving data transmission capability of the network.

\section{Realization of Maintenance Training Simulation System}

\section{A. Realization of Hardware System}

Considering the differences in function of each subsystem, design versatility and the consistency of the system interconnection, control all electrical functions core subsystems were uniform model DSP architecture. Taking starting up electrical subsystem as an example, the basic principle of the hardware structure was shown in Figure 3. This subsystem included a DSP smallest hardware system, 
a power system, signal processing, power-driven, CAN bus communication hardware units. DSP-based digital transformation enabled each subsystem with a more complete ability to state control and status information acquisition, which provided the foundation to achieve fault settings and status simulation.

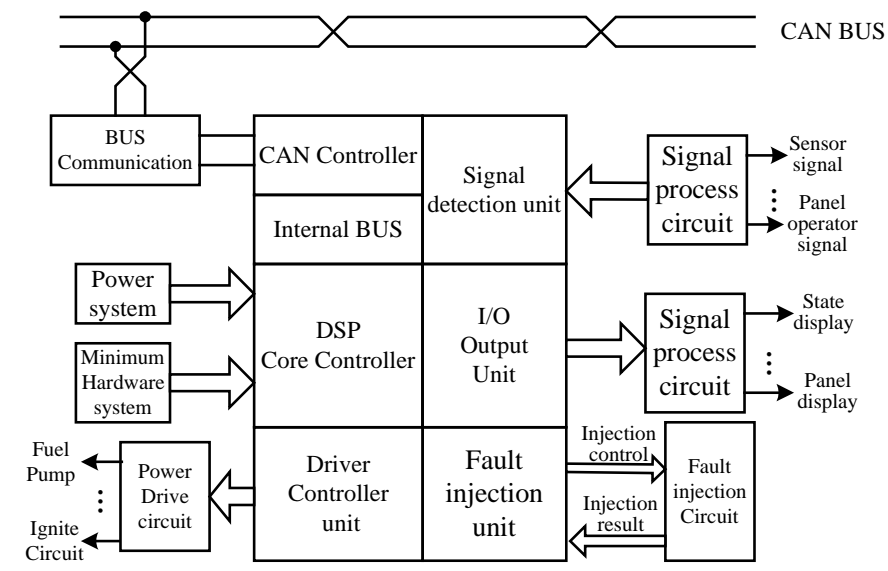

Figure 3. Hardware structure schematic diagram of the starting system

\section{B. Workflow of software System}

The Groovy scripting language was employed as software programming language. Display and Control Terminal system software initialized the CAN bus interface board, opening CAN bus, beginning scanning each subsystem, issuing handshake. If a response being get, which means that the subsystem being on-line, then the display and control terminal will establish CAN communication with the subsystem, and receiving and processing data; otherwise, it is determined that the subsystem offline. The modular design according to the specific circumstances of each subsystem was used, each subsystem software module including at least status display and faults set in two parts.

For the subsystem if being the on-line currently, it will continue to monitor whether there being data on the bus, after receiving the data, judgment data ID, if the ID number and the system ID is not the same, give up data, otherwise receiving data; after receiving the data subsystem compared them with the last received data, if the same two data showing no change in status or fault, without processing the data, if the two data are different, then there is a change status or fault, the data is sent to the display and control terminal status display interface, and processing fault control signals.

Display and control terminal operator by visualization window menus can set a variety of typical faults. after receiving the instruction to start the subsystem fault injection procedure, the relevant circuit automatically adjusts the voltage and current parameters, resulting in consistent with the actual fault phenomenon, trained accordingly personnel failure analysis and troubleshooting training.

\section{Conclusion}

In this paper, aiming at the instruction characteristic of maintenance training and support requirement, the novel simulation maintenance training system were designed based on digital redesign of aircraft engine electrical comments. The system was built with the core of integrated console, based on the CAN bus and the entire aircraft engine electrical digitized system. It can realize the control of each electrical system component and fault setting with the integrated console. At the same time, the maintenance training management terminal was developed based on Eclipse software, which providing environment-friendly software upgrade condition for the expansion of the system nodes and system improvements. Compared with traditional teaching methods, the designed training system can not only simulate the actual operation of electrical systems, and can simulate the engine consistent with real equipment failure phenomenon, so that students in the theoretical teaching closer to mounting environment, the actual operation and troubleshooting training. The system can dramatically increase the efficiency of training, save training funds and improve the informatization 
level of support technology.

\section{References}

[1] Jiang Wenbo, Jin Ou, He Jianbiao. Research and Testing of Real-time scheme on Linux System. Computer Mearment \& Control [J].2008,16 (4):461-463.

[2] Robert Day.Shared future-Eclipse makes it easier to move between embedded operating systems and target platforms [J].IET Electric Systems and Software.Feb-March 2007,20-23.

[3] Zhang Z,Jiang M.Using Eclipse as A Tool-Integration Platform for Software Developmen [J]. IEEE Software.24 (2).2007,87-89.

[4] Cockburn B F.Tutorial on Semiconductor Memory Testing [J]. Journal of Electronic. Testing:Theory and Application,1994,5(4):321-336.

[5] YANG Fan, FAN D i n g, PENG Ka. Design of Real-Time Measurement and Control System for Aeroengine Test Rig Basedon CAN [J]. Mearment \& Control Technology,2013,11(32):72-75. 